\title{
MicroRNA-217 is involved in the progression of atherosclerosis through regulating inflammatory responses by targeting sirtuin 1
}

\author{
LIYUN ZHANG, JUAN CHEN, QIN HE, ZE CHAO, XUYONG LI and MANHUA CHEN \\ Department of Cardiology, Wuhan Central Hospital, Wuhan, Hubei 430015, P.R. China
}

Received September 6, 2018; Accepted June 12, 2019

DOI: $10.3892 / \mathrm{mmr} .2019 .10581$

\begin{abstract}
Atherosclerosis is a chronic inflammatory disease, and it is a global clinical problem. The development of new and effective therapeutic targets for atherosclerosis is necessary. A number of microRNAs (miRNAs) have been demonstrated to serve a crucial role in atherosclerosis. However, the role of miRNA (miR)-217 in atherosclerosis remains unclear. Therefore, the aim of the present study was to investigate the role and mechanism of miR-217 in atherosclerosis. The level of miR-217 was detected in the blood of patients with atherosclerosis using reverse transcription-quantitative PCR. THP-1 acute monocytic leukemia cells were treated with oxidized low-density lipoprotein (ox-LDL) to develop an atherosclerotic cell model of macrophages. The relationship between miR-217 and sirtuin 1 (SIRT1) was determined by TargetScan and dual luciferase reporter assay. Cell apoptosis was measured by flow cytometry. Production of pro-inflammatory factors and triglyceride (TG) and total cholesterol (TC) levels were also determined. The results demonstrated that miR-217 was significantly upregulated in atherosclerosis. SIRT1 was demonstrated to be a direct target of miR-217 and was downregulated in atherosclerosis. Downregulation of miR-217 significantly inhibited ox-LDL-induced TG and TC level increase, cell apoptosis and the upregulation of the pro-inflammatory factors tumor necrosis factor $\alpha$, interleukin (IL)-6 and IL-1 $\beta$. Additionally, the SIRT1/AMP-activated protein kinase $\alpha / \mathrm{NF}-\kappa \mathrm{B}$ pathway was at least partially involved in modulating the effects of miR-217 inhibition on THP-1 cells treated with ox-LDL. In addition, the effects of miR-217 downregulation on ox-LDL-treated THP-1 cells were eliminated by SIRT1 silencing. In conclusion, the results of the present study indicated that miR-217 downregulation may relieve atherosclerosis through the inhibition of macrophage apoptosis and inflammatory response by targeting SIRT1.
\end{abstract}

Correspondence to: Dr Manhua Chen, Department of Cardiology, Wuhan Central Hospital, 26 Shenli Road, Wuhan, Hubei 430015, P.R. China

E-mail: chenmh0918@163.com

Key words: atherosclerosis, microRNA-217, sirtuin 1, macrophages, apoptosis, inflammation

\section{Introduction}

Atherosclerosis is a chronic inflammatory disease of the arterial wall that can cause cardiovascular disease, such as stroke or myocardial infarction, and peripheral artery disease $(1,2)$. The pathogenesis of atherosclerotic lesion formation is a multistage process. Endothelial injury and lipid-loaded macrophages participate in the development of atherosclerotic plaques (3). Macrophages serve an important role in the development and progression of atherosclerosis. Macrophage apoptosis and apoptotic body scavenging failure result in atherosclerosis inflammation (4). Inflammation serves a crucial role in all stages of the development of atherosclerosis $(5,6)$. At present, atherosclerosis is a global clinical problem that faces enormous challenges. Therefore, the molecular mechanism of atherosclerosis needs to be studied in an effort to seek new and effective therapeutic targets.

MicroRNAs (miRNAs) are a group of small endogenous non-coding RNAs, 22 nucleotides long, that post-transcriptionally regulate gene expression by binding to the 3'-untranslated region (UTR) of target mRNAs (7-9). miRNAs serve critical roles in the regulation of a number of biological processes, including cell proliferation, differentiation and apoptosis (10-12). Studies have demonstrated that miRNAs serve a key role in the regulation of normal physiology and disease (13), including atherosclerosis (14). miRNA (miR)-217 has been studied in various types of cancer including liver cancer, gastric cancer, lung adenocarcinoma and acute myeloid leukemia (15-18). Previous studies have revealed upregulation of miR-217 in atherosclerosis $(19,20)$. However, whether miR-217 is involved in the development of atherosclerosis through the regulation of macrophages is still unclear.

Sirtuin 1 (SIRT1), an $\mathrm{NAD}^{+}$-dependent deacetylase, plays an important role in the regulation of apoptosis and inflammatory responses (21-25). A recent report indicated that the inhibition of SIRT1 may promote atherosclerosis (26). However, the relationship between miR-217 and SIRT1 in atherosclerosis remains unclear.

Lipid-loaded macrophages participate in the development of atherosclerotic plaques (27). Oxidized low-density lipoprotein (ox-LDL) triggers intracellular events that enhance pro-inflammatory cytokines expression, resulting in the apoptosis of macrophages (28). Macrophage apoptosis and failure to clear apoptotic bodies may lead to atherosclerosis 
and inflammation (4). ox-LDL treated macrophages have been widely used to study atherosclerosis in vitro $(29,30)$.

The aim of the present study was to investigate the role of miR-217 in atherosclerosis using an in vitro cellular model induced by ox-LDL.

\section{Materials and methods}

Clinical samples. A total of 60 peripheral blood samples ( $2 \mathrm{ml}$ per individual) from 60 patients with atherosclerosis (age range, 47-78; male:female, 2:1), as well as 60 peripheral blood samples from 60 healthy volunteers (age range, 45-79; male:female, 2:1) were collected at Wuhan Central Hospital (Wuhan, China) between April 2016 and April 2017. Clinicopathological characteristics of patients were shown in Table I. The protocols were approved by the Ethics Committee of Wuhan Central Hospital and informed consent was obtained from each patient.

Cell culture and atherosclerotic cell model. The human acute monocytic leukemia cell line THP-1 was purchased from the American Type Culture Collection (cat. no. TIB-202). Cells were cultured in RPMI-1640 medium (Gibco; Thermo Fisher Scientific, Inc.) containing 10\% FBS (Gibco; Thermo Fisher Scientific, Inc.) and $1 \%$ penicillin/streptomycin mixed solution. Cells were incubated at $37^{\circ} \mathrm{C}$ with $5 \% \mathrm{CO}_{2}$.

To differentiate THP-1 monocytes into macrophages, THP-1 monocytes were treated with $10 \mathrm{nM}$ phorbol 12-myristate 13-acetate (Sigma-Aldrich; Merck KGaA) for $48 \mathrm{~h}$ (31). Atherosclerotic cell model of macrophages was established as previously described by treating differentiated THP-1 cells with $25 \mu \mathrm{g} / \mathrm{ml}$ ox-LDL for $24 \mathrm{~h}$ (31); the significant formation of THP-1 foam cells suggested that the model was successfully established.

Cell transfection. miR-217 inhibitor (5'-UACUGCAUC AGGAACUGAUUGGA-3'; Shanghai GenePharma Co., Ltd.), inhibitor control (5'-GCCUCCGGCUUCGCACCU CU-3'; Shanghai GenePharma Co., Ltd.), control-siRNA (cat. no. sc-36869; Santa Cruz Biotechnology, Inc.), SIRT1-siRNA (cat. no. sc-40986; Santa Cruz Biotechnology, Inc.) or miR-217 inhibitor + SIRT1-siRNA was transfected into THP-1 macrophages (ox-LDL untreated) using Lipofectamine ${ }^{\circledR} 2000$ reagent (Invitrogen; Thermo Fisher Scientific, Inc.) following the manufacturer's protocol at $37^{\circ} \mathrm{C}$ for $48 \mathrm{~h}$. Transfection efficiency was measured at $48 \mathrm{~h}$ post-transfection using reverse transcription-quantitative PCR (RT-qPCR).

Dual luciferase reporter assay. TargetScan bioinformatics software (www.targetscan.org/vert_71) was used to predict the potential targets of miR-217, and results identified binding sites between miR-217 and 3'UTR of sirtuin 1 (SIRT1). Dual luciferase reporter assay was performed to determine whether miR-217 directly binds to SIRT1. Wild-type (WT-SIRT1) and mutant (MUT-SIRT1) 3'UTR of SIRT1 were cloned into pmiR-RB-Report $^{\mathrm{TM}}$ dual luciferase reporter gene plasmid vectors (Guangzhou RiboBio Co., Ltd.) following the manufacturer's instructions. THP-1 cells were co-transfected with WT-SIRT1 or MUT-SIRT1 and miR-217 mimic or mimic control using Lipofectamine ${ }^{\circledR} 2000$ (Invitrogen; Thermo Fisher Scientific, Inc.) at $37^{\circ} \mathrm{C}$ for $48 \mathrm{~h}$. At $48 \mathrm{~h}$ post-transfection, luciferase activity was assessed by the Dual-luciferase Assay System (Promega Corporation) and normalized to Renilla luciferase activity.

Triglyceride (TG) and total cholesterol (TC) assays. The levels of TG and TC in the cell lysates of THP-1 macrophages were determined using the TG Assay kit (cat. no. ab65336; Abcam) and Cholesterol Assay kit (cat. no. ab133116; Abcam) following the manufacturer's instructions.

Apoptosis assay. Transfected and ox-LDL-treated THP-1 cells were used in the apoptosis assay. The Annexin V-FITC/propidium iodide (PI) apoptosis detection kit (cat. no. 70-AP101-100; Hangzhou MultiSciences Biotech Co., Ltd.) was used to analyze apoptotic rates. Briefly, cells ( $1 \times 10^{6}$ cells) were dyed with $5 \mu \mathrm{l}$ Annexin V-FITC and $5 \mu \mathrm{l}$ PI for $30 \mathrm{~min}$ at room temperature in the dark. Apoptotic rates were analyzed using a flow cytometer (BD Biosciences) with FlowJo software (version 7.6.1; FlowJo LLC).

ELISA. The levels of the tumor necrosis factor $\alpha$ (TNF- $\alpha)$ (cat no. PT518), interleukin (IL)-6 (cat no. PT330) and IL-1 $\beta$ (cat no. PT305) in the supernatant $(100 \mu \mathrm{l})$ of transfected and ox-LDL-treated THP-1 cells were determined using sandwich ELISA kits from Beyotime Institute of Biotechnology following the manufacturer's protocol of each kit. Cell supernatant was collected through centrifugation (500 g, $5 \mathrm{~min}$ ).

$R T-q P C R$. Total RNA from blood samples $(1 \mathrm{ml})$ and cells $\left(1 \times 10^{6}\right.$ cells) was extracted using TRIzol ${ }^{\circledR}$ reagent (Invitrogen; Thermo Fisher Scientific, Inc.) following the manufacturer's instructions. For cDNA generation, reverse transcription was performed using TaqMan MicroRNA Reverse Transcription kit (Applied Biosystems; Thermo Fisher Scientific, Inc.). cDNAs were analyzed by qPCR assay with SYBR Premix Ex Taq ${ }^{\mathrm{TM}}$ II (TliRNaseH Plus) kit (Takara Bio, Inc.). U6 for miRNA and GAPDH for mRNA were used as internal controls. Primer sequences for qPCR were as follows: miR-217, forward 5'-TACTGCATCAGG AACTGACTGGA-3', reverse 5'-GTGCAGGGTCCGAGG T-3'; SIRT1, forward 5'-AATCCAGTCATTAAAGGTCTA CAA-3', reverse 5'-TAGGACCATTACTGCCAGAGG-3'; U6, forward 5'-GCTTCGGCAGCACATATACTAAAAT-3', reverse 5'-CGCTTCACGAATTTGCGTGTCAT-3'; GAPDH, forward 5'-CTTTGGTATCGTGGAAGGACTC-3', reverse 5'-GTAGAGGCAGGGATGATGTTCT-3'. Expression levels were normalized to the respective internal controls and calculated using the $2^{-\Delta \Delta \mathrm{Cq}}$ method (32).

Western blot assay. Proteins from blood samples $(1 \mathrm{ml})$ and cells $\left(1 \times 10^{6}\right.$ cells) were extracted by using RIPA lysis buffer (cat. no. P0013E; Beyotime Institute of Biotechnology) following the manufacturer's instructions. Bicinchoninic acid assay kit (Pierce; Thermo Fisher Scientific; Inc.) was used to quantify the protein samples. Equal amounts of protein samples (30 $\mu \mathrm{g} / \mathrm{lane})$ were separated on $12 \%$ SDS-PAGE and transferred onto PVDF membranes (Merck KGaA). Following blocking in 5\% skimmed milk at room temperature for $1.5 \mathrm{~h}$, the membranes were 
Table I. Clinical characteristic of atherosclerotic patients and healthy controls.

\begin{tabular}{lcc}
\hline Variables & Patients $(\mathrm{n}=60)$ & Controls $(\mathrm{n}=60)$ \\
\hline Age (range, years) & $47-78$ & $45-79$ \\
Sex & & \\
$\quad$ Male & 40 & 40 \\
$\quad$ Female & 20 & 20 \\
Stable CAD for $\geq 4$ months & 60 & 0 \\
Hypertension & 45 & 0 \\
Diabetes mellitus & 32 & 0 \\
Active smoker & 28 & 11 \\
Obesity $\left(\right.$ BMI $\left.>25 \mathrm{~kg} / \mathrm{m}^{2}\right)$ & 13 & 2 \\
Total cholesterol $(\mathrm{mmol} / \mathrm{l})$ & $5.32 \pm 0.81$ & $4.61 \pm 0.45$ \\
LDL cholesterol $(\mathrm{mmol} / \mathrm{l})$ & $3.79 \pm 0.39$ & $2.80 \pm 0.45$ \\
HDL cholesterol $(\mathrm{mmol} / \mathrm{l})$ & $1.19 \pm 0.52$ & $1.20 \pm 0.30$ \\
Triglycerides $(\mathrm{mmol} / \mathrm{l})$ & $1.83 \pm 0.42$ & $1.37 \pm 0.64$ \\
\hline
\end{tabular}

BMI, body mass index; CAD, coronary artery disease; HDL, high density lipoprotein; LDL, low density lipoprotein.

incubated with primary antibodies against SIRT1 (120 kDa; 1:1,000; cat. no. 9475; Cell Signaling Technology, Inc.), phosphorylated (p)-AMP-activated protein kinase $\alpha$ (AMPK- $\alpha$; 62 kDa; 1:1,000; cat. no. 50081; Cell Signaling Technology, Inc.), AMPK- $\alpha$ (62 kDa; 1:1,000; cat. no. 5831; Cell Signaling Technology, Inc.), p-NF-kB p65 (65 kDa; 1:1,000; cat. no. 3033; Cell Signaling Technology, Inc.), p65 (65 kDa; 1:1,000; cat. no. 8242; Cell Signaling Technology, Inc.) and $\beta$-actin (45 kDa; 1:1,000; cat. no. 4970; Cell Signaling Technology, Inc.) overnight at $4^{\circ} \mathrm{C}$. Subsequently, the membranes were incubated with the horseradish peroxidase-conjugated goat anti-rabbit immunoglobulin G secondary antibody (1:2,000; cat. no. 7074; Cell Signaling Technology, Inc.) at room temperature for $2 \mathrm{~h}$. Protein bands were visualized using chemiluminescent ECL reagent (EMD Millipore) and quantified by densitometry (QuantityOne 4.5.0 software; Bio-Rad Laboratories Inc.). Expression was normalized to $\beta$-actin.

Statistical analysis. Data are presented as the mean \pm SD. SPSS 17.0 software (SPSS, Inc.) was used to perform statistical analysis. Differences between groups were determined using Student's t-test or one-way analysis of variance with a Bonferroni post hoc test. $\mathrm{P}<0.05$ was considered to indicate a statistically significant difference.

\section{Results}

miR-217 is upregulated in atherosclerosis. Expression levels of miR-217 were determined in the blood of 60 patients with atherosclerosis and 60 healthy volunteers using RT-qPCR. The results indicated that, compared with healthy volunteers, the level of miR-217 in the blood of patients with atherosclerosis was significantly increased (Fig. 1A). In addition, the level of miR-217 was significantly increased in ox-LDL-treated THP-1 macrophages (atherosclerosis model) compared with untreated
THP-1 macrophages (Fig. 1B). These data suggested that miR-217 may be upregulated in atherosclerosis.

SIRT1 is a target of miR-217. TargetScan identified SIRT1 as a potential miR-217 target (Fig. 2A). Results from the dual-luciferase reporter assay demonstrated that luciferase activity was significantly reduced in THP-1 macrophages co-transfected with SIRT1-WT reporter plasmids and miR-217 mimic compared with SIRT1-WT and mimic control; no differences were observed between co-transfections with SIRT1-MUT reporter plasmids and miR-217 mimic or mimic control (Fig. 2B). miR-217 significantly enhanced the level of miR-217 in THP-1 macrophages (Fig. 2C). These data indicated that miR-217 may directly target SIRT1.

SIRT1 is downregulated in atherosclerosis. SIRT1 mRNA expression levels were examined in the blood of 60 patients with atherosclerosis and 60 healthy volunteers using RT-qPCR. Compared with the healthy volunteers, SIRT1 mRNA expression level in the blood of patients with atherosclerosis was significantly decreased compared with healthy volunteers (Fig. 3A). In addition, ox-LDL treatment significantly decreased SIRT1 mRNA level in THP-1 macrophages compared with untreated cells (Fig. 3B). ox-LDL treatment markedly decreased SIRT1 protein expression in THP-1 macrophages compared with the untreated cells (Fig. 3C). These data suggested that SIRT1 may be downregulated in atherosclerosis.

miR-217 downregulation reduces TG and TC levels triggered by $o x-L D L$. To determine the role of miR-217 in atherosclerosis, miR-217 inhibitor, inhibitor control, control-siRNA, SIRT1-siRNA or miR-217 inhibitor + SIRT1-siRNA was transfected into THP-1 macrophages for $24 \mathrm{~h}$ prior to treatment with $25 \mu \mathrm{g} / \mathrm{ml}$ ox-LDL for $24 \mathrm{~h}$. Transfection efficiency was determined by RT-qPCR and/or western blot analysis. miR-217 inhibitor significantly downregulated the miR-217 level in THP-1 macrophages compared with the untreated cells (Fig. 4A), and SIRT1-siRNA significantly decreased the SIRT1 mRNA level in THP-1 macrophages compared with untreated cells (Fig. 4B). The protein level of SIRT1 was also reduced in THP-1 macrophages by SIRT1-siRNA treatment (Fig. 4C). Additionally, miR-217 inhibitor significantly increased the mRNA levels of SIRT1, and the enhancement was reversed by SIRT1-siRNA (Fig. 4D). The miR-217 inhibitor markedly enhanced the expression of SIRT1, and this was reversed by SIRT1-siRNA (Fig. 4E).

The effect of miR-217 on TG and TC levels in the atherosclerosis cell model was also determined. Compared with the untreated control group, $25 \mu \mathrm{g} / \mathrm{ml}$ ox-LDL treatment significantly enhanced the levels of TG and TC, whereas miR-217 inhibitor significantly reduced TG and TC levels induced by ox-LDL (Fig. 5). In addition, co-treatment with SIRT1-siRNA reversed the effects of miR-217 inhibitor on the levels of TG and TC in THP-1 macrophages (Fig. 5).

miR-217 downregulation inhibits ox-LDL-induced apoptosis. The effect of miR-217 on THP-1 cell apoptosis was analyzed. Ox-LDL treatment significantly induced THP-1 macrophage apoptosis (Fig. 6), which was notably inhibited by miR-217 inhibitor transfection, and this inhibition was reversed by co-transfection with SIRT1-siRNA. 

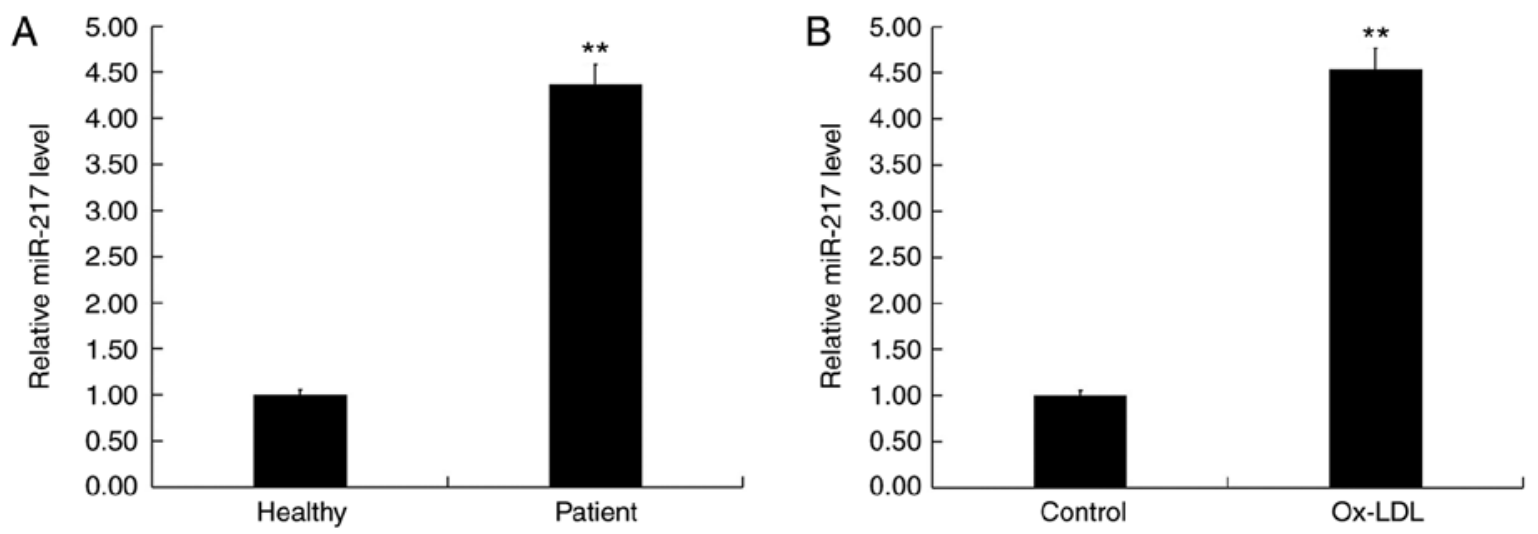

Figure 1. miR-217 expression levels in atherosclerosis. (A) miR-217 expression levels in the blood of 60 patients with atherosclerosis and 60 healthy volunteers was detected using RT-qPCR. (B) THP-1 macrophage cells were treated with $25 \mu \mathrm{g} / \mathrm{ml}$ ox-LDL for $24 \mathrm{~h}$, and the level of miR-217 in THP-1 cells was detected using RT-qPCR. ${ }^{* *} \mathrm{P}<0.01$ vs. Healthy patients or Control cells. miR-217, microRNA-217; ox-LDL, oxidized low-density lipoprotein; RT-qPCR, reverse transcription-quantitative PCR.

A Position 1519-1526 of SIRT1 3' UTR 5' .... UGGCUACACUAagGaAlGCAGUA. hsa-miR-217 3' agguagucaagGacuacGicau

B

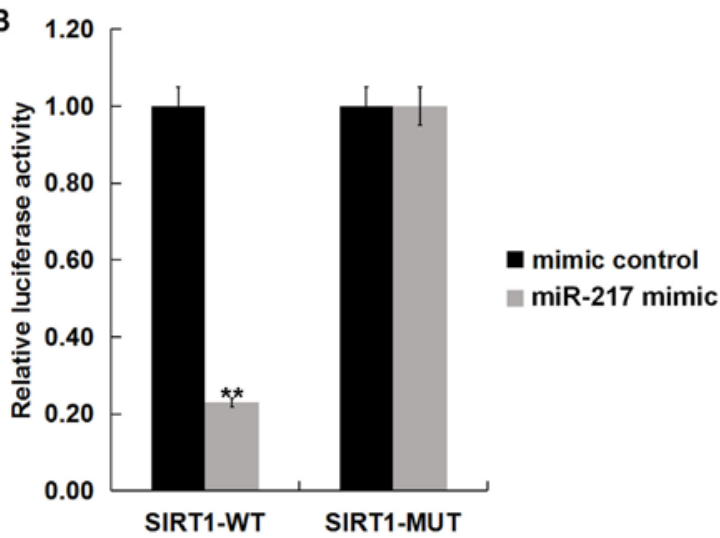

C

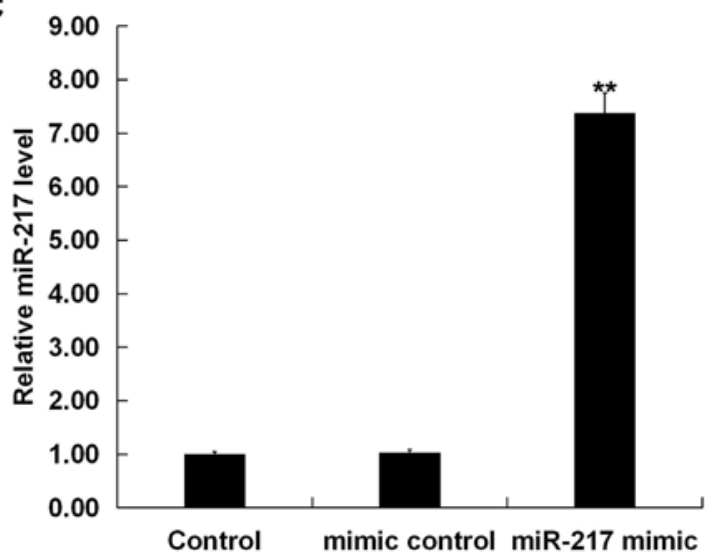

Figure 2. SIRT1 is a direct target of miR-217. (A) miR-217 target site in the 3'UTR of SIRT1 was predicted using TargetScan. (B) Luciferase activity of a reporter containing WT-SIRT1 3'UTR or MUT-SIRT1 3'UTR co-transfected with miR-217 mimic or control. "** $\mathrm{P}<0.01$ vs. Mimic control. (C) THP-1 macrophage cells were transfected with mimic control or miR-217 mimic for $48 \mathrm{~h}$ and the level of miR-217 was determined using reverse transcription-quantitative-PCR ${ }^{* *} \mathrm{P}<0.01$ vs. control. miR-217, microRNA-217; MUT, mutant; SIRT1, sirtuin 1; UTR, untranslated region; WT, wild-type.

A

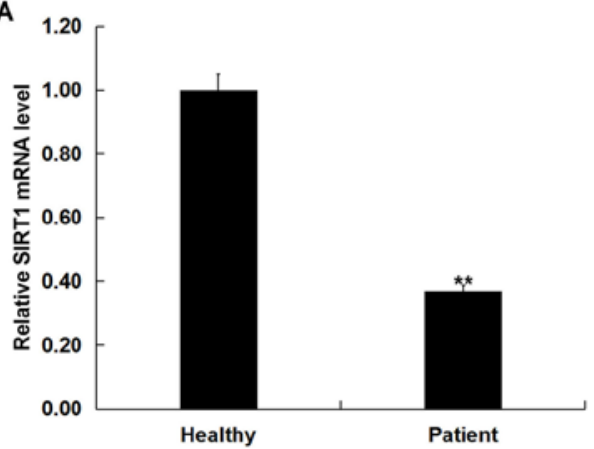

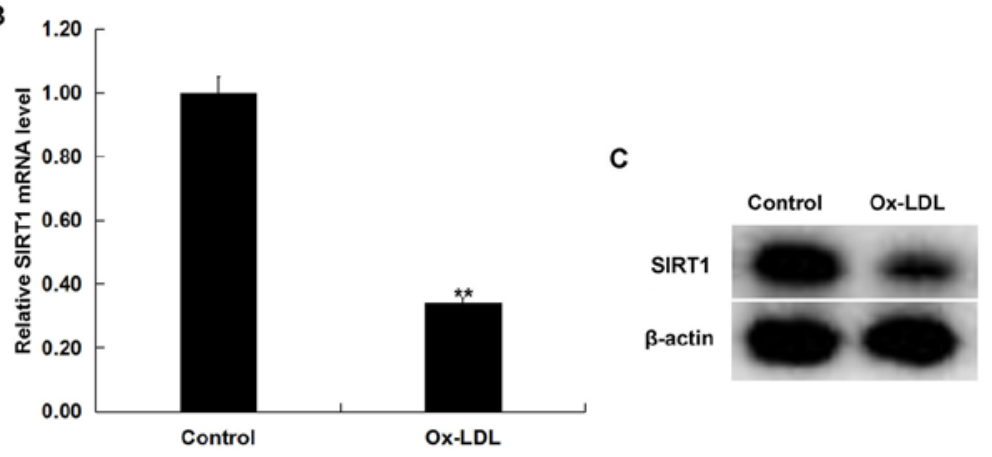

Figure 3. SIRT1 expression levels in atherosclerosis. (A) SIRT1 mRNA expression levels in the blood of 60 patients with atherosclerosis and 60 healthy volunteers was detected using RT-qPCR. (B and C) THP-1 macrophages were treated with $25 \mu \mathrm{g} / \mathrm{ml}$ ox-LDL for $24 \mathrm{~h}$, and SIRT1 (B) mRNA and (C) protein expression levels in THP-1 macrophages were detected using RT-qPCR and western blotting, respectively. ${ }^{* *} \mathrm{P}<0.01$ vs. Healthy patients or Control cells. ox-LDL, oxidized low-density lipoprotein; RT-qPCR, reverse transcription-quantitative PCR; SIRT1, sirtuin 1.

miR-217 downregulation inhibits ox-LDL-induced inflammatory response. The levels of inflammatory factors were detected by ELISA. The results demonstrated that treatment with ox-LDL increased TNF- $\alpha$, IL- 6 , and IL-1 $\beta$ content in 
A

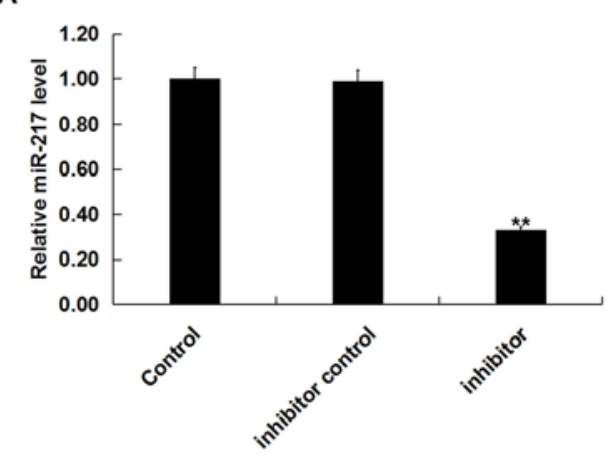

D

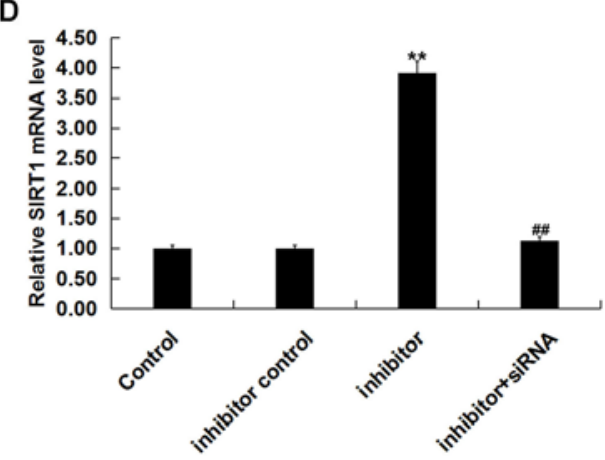

B

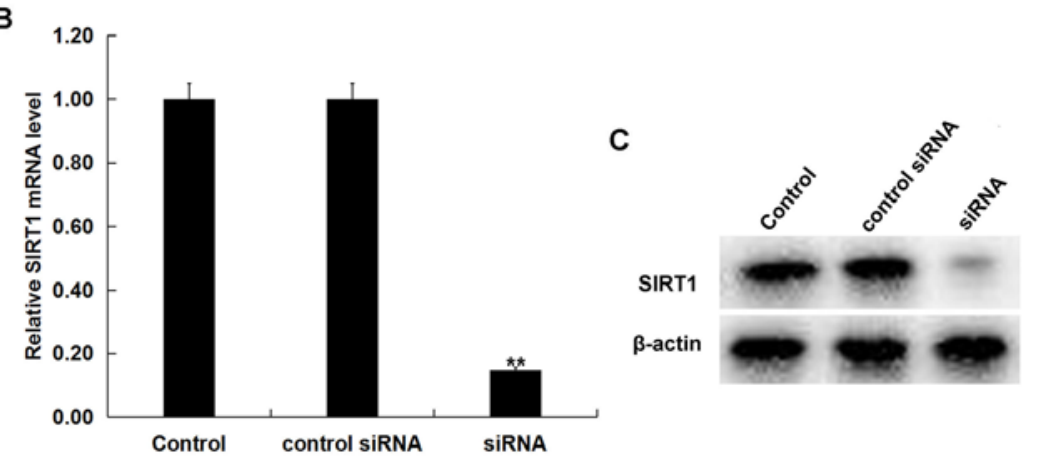

E

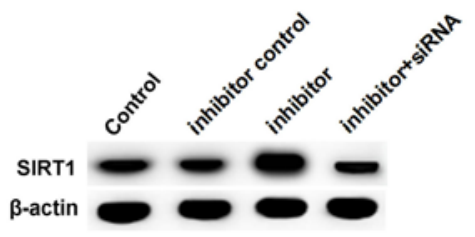

Figure 4. miR-217 inhibitor enhances SIRT1 expression in THP-1 macrophages. (A) Relative miR-217 expression level in THP-1 macrophages treated with miR-217 inhibitor. (B) Relative SIRT1 mRNA expression in THP-1 macrophages treated with SIRT1 siRNA. (C) SIRT1 protein expression in THP-1 macrophages treated with SIRT1 siRNA. (D) Relative SIRT1 mRNA expression and (E) protein expression in THP-1 macrophages treated with inhibitor control, miR-217 inhibitor or miR-217 inhibitor + SIRT1 siRNA. ${ }^{* *} \mathrm{P}<0.01$ vs. Untreated control group; ${ }^{\# \#} \mathrm{P}<0.01$ vs. Inhibitor. miR-217, microRNA-217; SIRT1, sirtuin 1 .
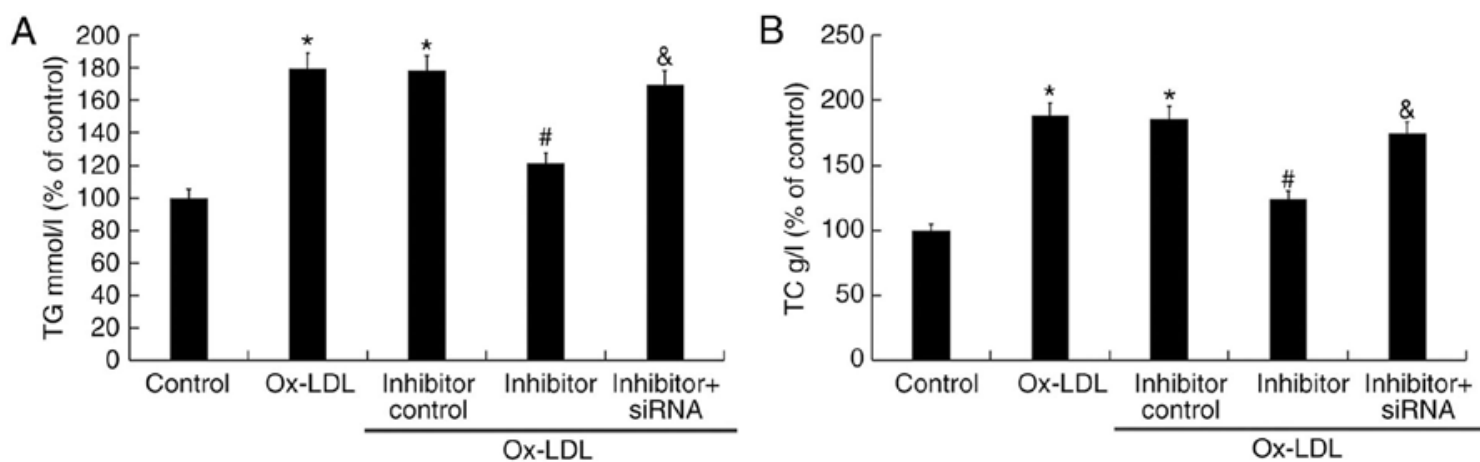

Figure 5. Effects of miR-217 inhibitor on TG and TC level in THP-1 macrophage cells. THP-1 cells were pre-transfected with miR-217 inhibitor, inhibitor control or miR-217 inhibitor + SIRT1-siRNA and subsequently treated ox-LDL. (A) TG and (B) TC levels in THP-1 macrophages were determined. ${ }^{*}<<0.05$ vs. Control; " $\mathrm{P}<0.05$ vs. ox-LDL; ${ }^{\circledR} \mathrm{P}<0.05$ vs. Inhibitor. ox-LDL, oxidized low-density lipoprotein; miR-217, microRNA-217; SIRT1, sirtuin 1 ; TC, total cholesterol; TG, triglyceride.

THP-1 macrophage culture, which was significantly decreased by miR-217 inhibitor transfection (Fig. 7). The effects of miR-217 inhibitor were reversed by SIRT1 silencing (Fig. 7).

Effects miR-217 downregulation on SIRT1/AMPK$\alpha / N F-\kappa B$ pathway in ox-LDL-treated THP-1 macrophages. The SIRT1/AMPK- $\alpha / \mathrm{NF}-\kappa \mathrm{B}$ pathway in ox-LDL-treated THP-1 macrophages was analyzed. Ox-LDL treatment markedly decreased SIRT1 protein level, reduced p-AMPK- $\alpha$ protein level, and increased the phosphorylation level of NF- $\kappa B$ p65 protein in THP-1 cells (Fig. 8A). Ox-LDL treatment significantly decreased the level of SIRT1 mRNA (Fig. 8B), reduced the p-AMPK- $\alpha /$ AMPK- $\alpha$ ratio (Fig. 8C), and increased the ratio of p-p65/p65 (Fig. 8D) in THP-1 cells. Compared with the ox-LDL-only treatment group, co-treatment with the miR-217 inhibitor markedly enhanced SIRT1 and p-AMPK- $\alpha$ expression, and reduced p-p65 protein level. SIRT1 silencing significantly reversed the effects of miR-217 inhibitor on the expression of SIRT1, p-AMPK- $\alpha$ and p-p65 in THP-1 macrophages.

\section{Discussion}

The results of the present study demonstrated that miR-217 was significantly upregulated in the blood of patients with atherosclerosis and ox-LDL-treated THP-1 macrophages. 

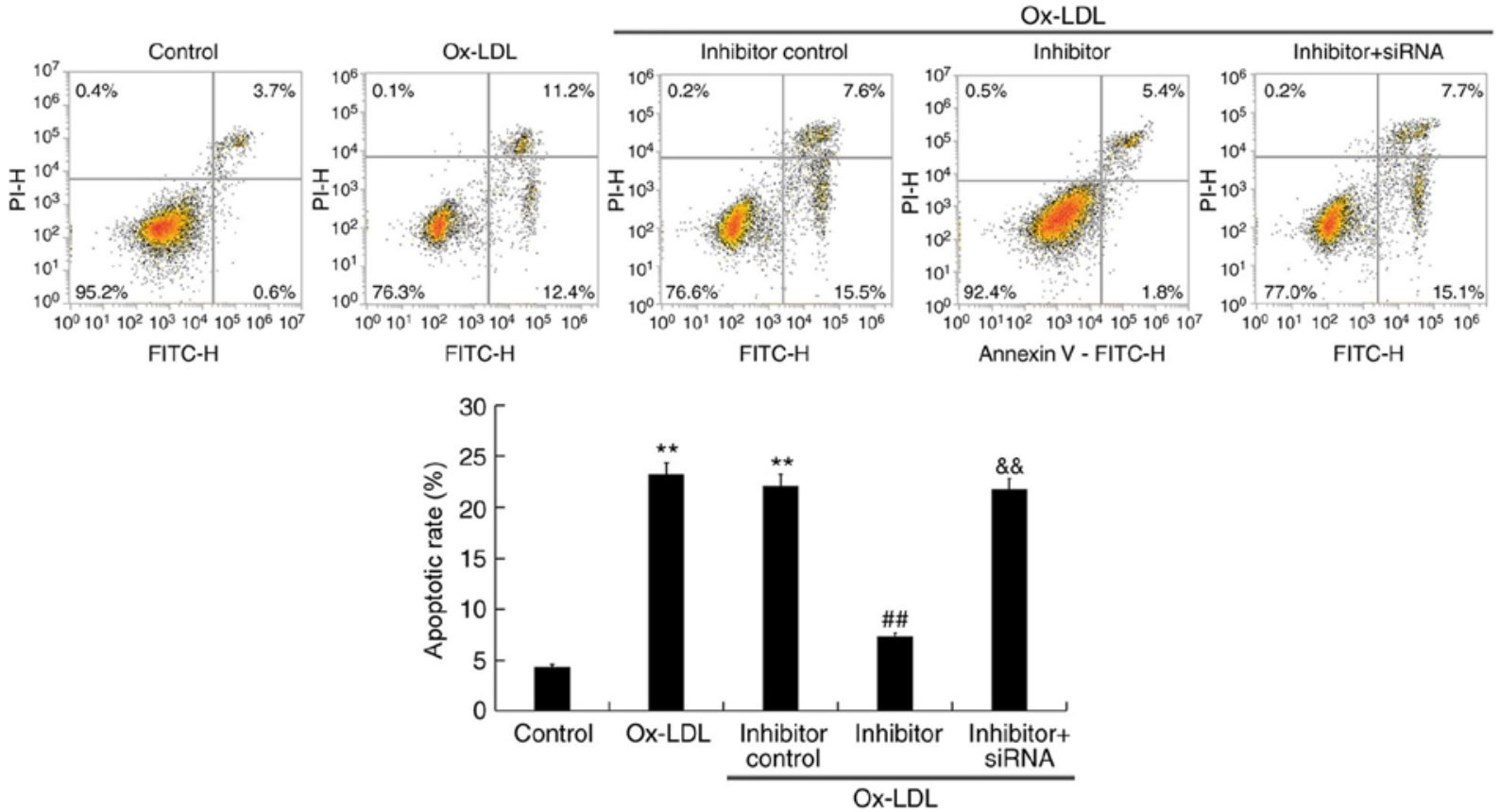

Figure 6. Effects of miR-217 inhibitor on THP-1 macrophage apoptosis. THP-1 cells were pre-transfected with miR-217 inhibitor, inhibitor control or miR-217 inhibitor+ SIRT1-siRNA and treated with ox-LDL. The level of apoptosis in the THP-1 macrophages was analyzed by flow cytometry. The early and late stages of apoptosis were examined. ${ }^{* *} \mathrm{P}<0.01$ vs. Control; ${ }^{\# \#} \mathrm{P}<0.01$ vs. ox-LDL; ${ }^{\& \&} \mathrm{P}<0.01$ vs. Inhibitor. ox-LDL, oxidized low-density lipoprotein; miR-217, microRNA-217; SIRT1, sirtuin 1.

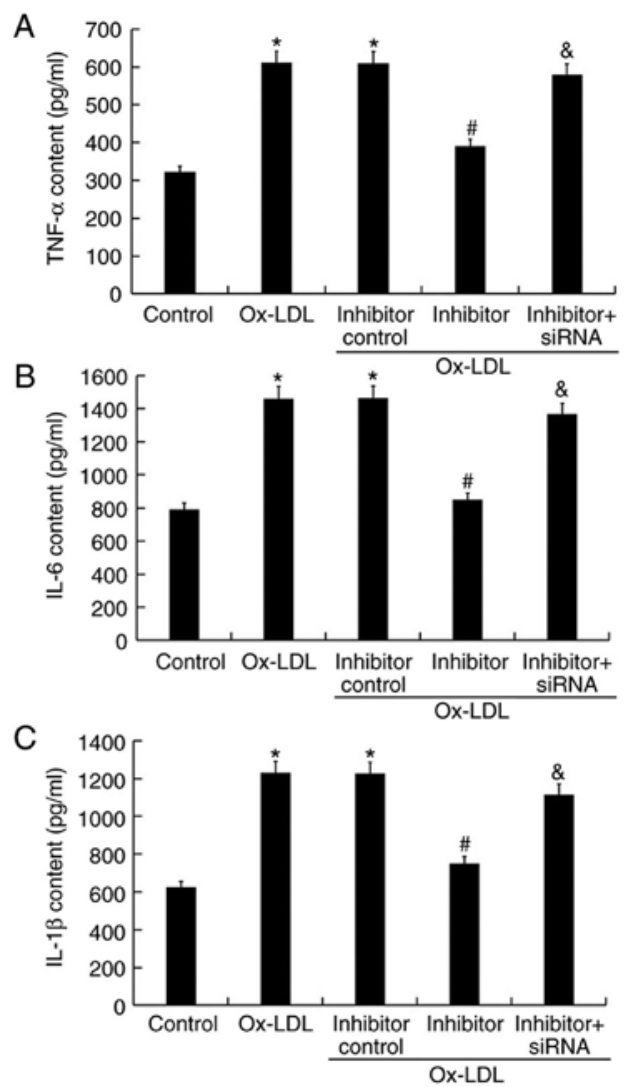

Figure 7. Effects of miR-217 inhibitor on the expression of inflammatory factors in THP-1 macrophages. THP-1 macrophages were pre-transfected with miR-217 inhibitor, inhibitor control, or miR-217 inhibitor + SIRT1-siRNA and treated with ox-LDL. The protein levels of (A) TNF- $\alpha$, (B) IL-6, and (C) IL-1 $\beta$ in the supernatant of the THP-1 macrophages were detected using ELISA. ${ }^{*} \mathrm{P}<0.05$ vs. Control; ${ }^{*} \mathrm{P}<0.05$ vs. ox-LDL group; ${ }^{\text {}} \mathrm{P}<0.05$ vs. Inhibitor. ox-LDL, oxidized low-density lipoprotein; miR-217, microRNA-217; SIRT1, Sirtuin-1.
SIRT1 was demonstrated to be a direct target of miR-217, and it was downregulated in the blood of patients with atherosclerosis and ox-LDL-treated THP-1 macrophages. Further analysis indicated that ox-LDL-induced apoptosis and TNF- $\alpha$, IL-1 $\beta$ and IL-6 expression levels in THP-1 macrophages were repressed by miR-217 inhibitor transfection. The results of this study suggested that the effects of miR-217 inhibitor on ox-LDL-treated THP-1 macrophages were eliminated by SIRT1 silencing with siRNA. The data obtained in the current study supported the hypothesis that miR-217/SIRT1 axis may be a novel therapeutic target for treating atherosclerosis.

Atherosclerosis is one of the leading causes of morbidity and mortality in the world, and it is a major threat worldwide (33). Therefore, finding new targets for the treatment of atherosclerosis is of great importance. The key cell events of atherosclerosis include hyperlipidemia, monocyte recruitment, differentiation into macrophages, foam cell formation and induced inflammation (34). A previous study suggested that inflammation drives the formation, development and rupture of atherosclerotic plaques (35). Therefore, the study of potential targets for atherosclerotic inflammatory processes may provide new therapeutic strategies for atherosclerosis (36). Previous studies have also demonstrated the roles of miRNAs in the pathogenesis and development of atherosclerosis. Li et al reported that miR-30c-5p inhibits endothelial cell pyroptosis by inhibiting forkhead box $\mathrm{O} 3$ in atherosclerosis (37). Wei et al reported that $\mathrm{miR}-342-5 \mathrm{p}$ promotes inflammatory macrophage activation during atherosclerosis (38). Additionally, miR-126, miR-150, miR-155 and miR-142-3p have been demonstrated to serve critical roles in the development of atherosclerosis (39-42). miR-217, which has been widely studied in cancer (15-18), has been revealed to be upregulated in atherosclerosis $(19,20)$. However, the role and molecular mechanism 


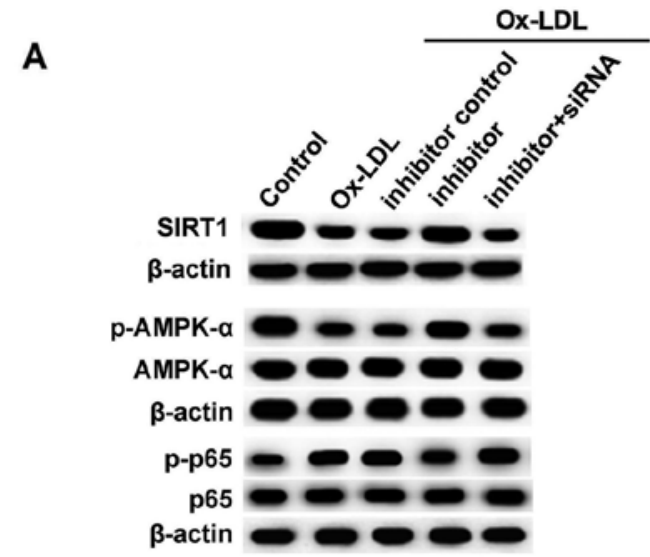

C

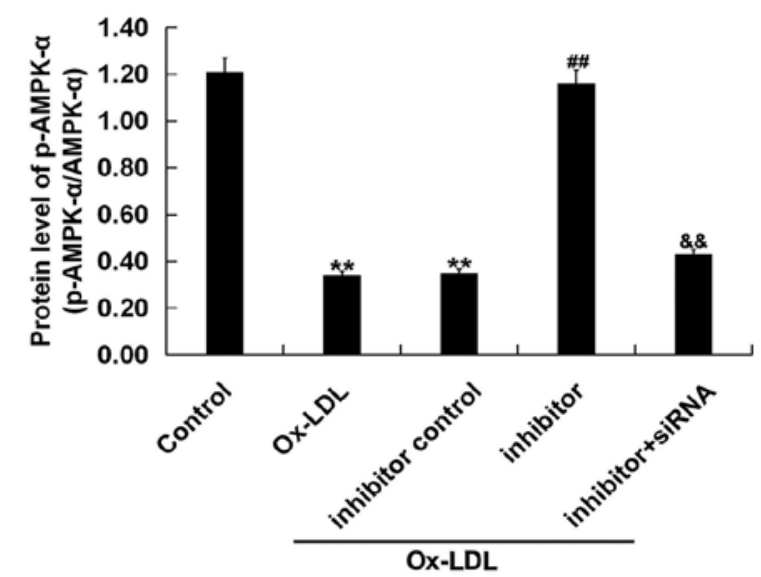

B
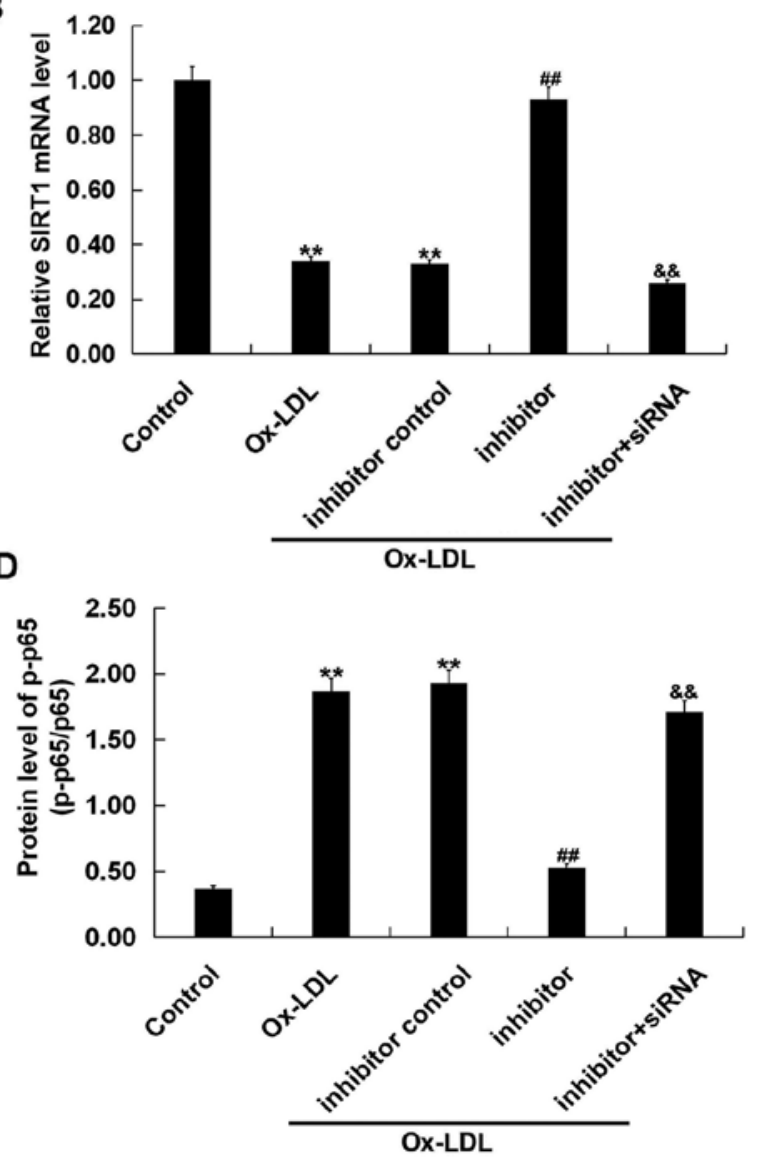

Figure 8. Effects of miR-217 inhibitor on SIRT1/AMPK- $\alpha$ NF- $\kappa$ B pathway in THP-1 macrophages. THP-1 macrophages were pre-transfected with miR-217 inhibitor, inhibitor control, or miR-217 inhibitor + SIRT1-siRNA and treated with ox-LDL. (A) The protein levels of SIRT1, p-AMPK- $\alpha$, AMPK- $\alpha$, p-p65, and p65 in THP-1 macrophages was determined using western blotting. (B) the mRNA level of SIRT1 in THP-1 macrophages was determined using reverse transcription-quantitative PCR. The ratio of (C) p-AMPK- $\alpha /$ AMPK- $\alpha$ and (D) p-p65/p65 was calculated. ${ }^{* *} \mathrm{P}<0.01$ vs. Control; ${ }^{\# \#} \mathrm{P}<0.01 \mathrm{vs}$. ox-LDL; ${ }^{\&} \mathrm{P}<0.01$ vs. Inhibitor. AMPK- $\alpha, 5$ '-AMP-activated kinase $\alpha$; miR-217, microRNA-217; ox-LDL, oxidized low density lipoprotein; p, phosphorylated; SIRT1, sirtuin 1.

of miR-217 in atherosclerosis remain largely unknown. The present study aimed to determine whether miR-217 is involved in the development of atherosclerosis through the regulation of macrophages.

SIRT1, an $\mathrm{NAD}^{+}$-dependent deacetylase, is involved in apoptosis (21) and serves important roles in the regulation of inflammatory responses (22-25). For example, SIRT1 protein levels are downregulated by IL $1 \beta / \mathrm{NF} \kappa \mathrm{B}$ signaling in acetaminophen hepatotoxicity, resulting in inflammation and oxidative stress (23). The inhibition of SIRT1 leads to oxidative stress and inflammation in patients with coronary artery disease (24). Wu et al reported that SIRT1 is involved in the invasion and metastasis of human esophageal cancer cells by inducing epithelial mesenchymal transition through the regulation of Snail expression (43). Yang et al demonstrated that SIRT1 inhibition promotes atherosclerosis through impaired autophagy (26). These results indicated that miR-217 may serve an important role in the development of atherosclerosis though the regulation of apoptosis and inflammatory responses in macrophages by regulating the expression of SIRT1.

To explore the potential function of miR-217 in atherosclerosis, loss-of-function experiments were performed using miR-217 inhibitor. miR-217 downregulation significantly inhibited the ox-LDL-induced increase of TG and TC levels, apoptosis and the upregulation of pro-inflammatory factors (TNF- $\alpha$, IL-6, and IL-1 $\beta$ ) in THP-1 macrophages. AMPK $\alpha /$ SIRT1 pathway activation may inhibit NF- $\kappa$ B-related inflammation (44). AMPK activation mediates vascular inflammation and leukocyte adhesiveness (45). Therefore, AMPK may have potential roles in cardiovascular disease through its anti-inflammatory effect (46). To explore the molecular mechanism of the effect of miR-217 inhibitor on ox-LDL treated THP-1 macrophages, the SIRT1/AMPK- $\alpha / \mathrm{NF}-\kappa \mathrm{B}$ pathway was analyzed in the present study. The results indicated that ox-LDL treatment significantly inhibited AMPK $\alpha /$ SIRT1 pathway activation, promoting $\mathrm{NF}-\kappa \mathrm{B}$ pathway activation, which could lead to an inflammatory response in THP-1 macrophages; the effects of the miR-217 inhibitor on ox-LDL treated THP-1 macrophages were eliminated by SIRT1 silencing.

In conclusion, the results of the present study demonstrated that miR-217 was upregulated in atherosclerosis, and its inhibition may relieve atherosclerosis through inhibiting macrophage apoptosis and inflammation response by restoring SIRT1 expression. miR-217 may be a potential therapeutic target for the treatment of atherosclerosis. However, the present study is only a preliminary study of the role of 
miR-217 in atherosclerosis; more experimental research is needed to confirm the current results. For instance, the role of SIRT1 alone in atherosclerosis should be investigated. The association between miR-217/SIRT1 and the clinicopathological characteristics of patients with atherosclerosis should be explored. Additionally, miR-217/SIRT1 in atherosclerosis should be studied in vivo.

\section{Acknowledgements}

Not applicable.

\section{Funding}

No funding was received.

\section{Availability of data and materials}

The analyzed data sets generated during the present study are available from the corresponding author on reasonable request.

\section{Authors' contributions}

LZ contributed to study design, data collection, statistical analysis, data interpretation and manuscript preparation. JC, $\mathrm{QH}, \mathrm{ZC}, \mathrm{XL}$ and MC contributed to data collection, statistical analysis.

\section{Ethics approval and consent to participate}

The protocols were approved by the Ethics Committee of Wuhan Central Hospital (Wuhan, China), and informed consent was obtained from each patient.

\section{Consent for publication}

Not applicable.

\section{Competing interests}

The authors declare that they have no competing interest.

\section{References}

1. Libby P and Theroux P: Pathophysiology of coronary artery disease. Circulation 111: 3481-3488, 2005.

2. Williams KJ and Tabas I: Atherosclerosis and inflammation. Science 297: 521-522, 2002.

3. Ross R: Atherosclerosis-an inflammatory disease. N Engl J Med 340: 115-126, 1999.

4. Tabas I: Macrophage death and defective inflammation resolution in atherosclerosis. Nat Rev Immunol 10: 36-46, 2010.

5. Hansson GK and Libby P: The immune response in atherosclerosis: A double-edged sword. Nat Rev Immunol 6: 508-519, 2006.

6. Hansson GK and Hermansson A: The immune system in atherosclerosis. Nat Immunol 12: 204-212, 2011.

7. Bartel DP: MicroRNAs: Genomics, biogenesis, mechanism, and function. Cell 116: 281-297, 2004.

8. Hammond SM: An overview of microRNAs. Adv Drug Deliv Rev 87: 3-14, 2015.

9. Ghildiyal $M$ and Zamore PD: Small silencing RNAs: An expanding universe. Nat Rev Genet 10: 94-108, 2009.

10. Soifer HS, Rossi JJ and Saetrom P: MicroRNAs in disease and potential therapeutic applications. Mol Ther 15: 2070-2079, 2017.
11. Krol J, Loedige I and Filipowicz W: The widespread regulation of microRNA biogenesis, function and decay. Nat Rev Genet 11: 597-610, 2010.

12. O'Connell RM, Rao DS, Chaudhuri AA and Baltimore D: Physiological and pathological roles for microRNAs in the immune system. Nat Rev Immunol 10: 111-122, 2010.

13. Mendell JT and Olson EN: MicroRNAs in stress signaling and human disease. Cell 148: 1172-1187, 2012.

14. Hosin AA, Prasad A, Viiri LE, Davies AH and Shalhoub J: MicroRNAs in atherosclerosis. J Vasc Res 51: 338-3349, 2014.

15. Wang LP, Wang JP and Wang XP: HOTAIR contributes to the growth of liver cancer via targeting miR-217. Oncol Lett 15: 7963-7972, 2018.

16. Safaralizadeh R, Ajami N, Nemati M, Hosseinpourfeizi M, Azimzadeh Isfanjani A and Moaddab SY: Dysregulation of miR-216a and miR-217 in gastric cancer and their clinical significance. J Gastrointest Cancer 50: 78-83, 2019.

17. Liu AN, Qu HJ, Yu CY and Sun P: Knockdown of LINC01614 inhibits lung adenocarcinoma cell progression by upregulating miR-217 and downregulating FOXP1. J Cell Mol Med 22: 4034-4044, 2018.

18. Yan J, Wu G, Chen J, Xiong L, Chen G and Li P: Downregulated miR-217 expression predicts a poor outcome in acute myeloid leukemia. Cancer Biomark 22: 73-78, 2018.

19. Liu H, Li X, Song Y and Wang Z: MicroRNA-217 attenuates intima-media complex thickness of ascending aorta measured by ultrasound bio-microscopy and inhibits inflammation and lipid metabolism in atherosclerotic models of $\mathrm{ApoE}^{-/-}$mice. Lipids Health Dis 17: 170, 2018.

20. Liu K, Xuekelati S, Zhou K, Yan Z, Yang X, Inayat A, Wu J and Guo X: Expression profiles of six atherosclerosis-associated microRNAs that cluster in patients with hyperhomocysteinemia: A clinical study. DNA Cell Biol 37: 189-198, 2018.

21. Nogueiras R, Habegger KM, Chaudhary N, Finan B, Banks AS, Dietrich MO, Horvath TL, Sinclair DA, Pfluger PT and Tschöp MH: Sirtuin 1 and sirtuin 3: Physiological modulators of metabolism. Physiol Rev 92: 1479-1514, 2012.

22. da Cunha MSB and Arruda SF: Tucum-do-Cerrado (Bactris setosa Mart.) may promote anti-aging effect by upregulating SIRT1-Nrf2 pathway and attenuating oxidative stress and inflammation. Nutrients 9: pii: E1243, 2017.

23. Rada P, Pardo V, Mobasher MA, García-Martínez I, Ruiz L, González-Rodríguez Á, Sanchez-Ramos C, Muntané J, Alemany S, James LP, et al: SIRT1 controls acetaminophen hepatotoxicity by modulating inflammation and oxidative stress. Antioxid Redox Signal 28: 1187-1208, 2018.

24. Chan SH, Hung CH, Shih JY, Chu PM, Cheng YH, Lin HC and Tsai KL: SIRT1 inhibition causes oxidative stress and inflammation in patients with coronary artery disease. Redox Biol 13: 301-309, 2017.

25. Cheng YY, Kao CL, Ma HI, Hung CH, Wang CT, Liu DH, Chen PY and Tsai KL: SIRT1-related inhibition of pro-inflammatory responses and oxidative stress are involved in the mechanism of nonspecific low back pain relief after exercise through modulation of Toll-like receptor 4. J Biochem 158: 299-308, 2015.

26. Yang X, Wei J, He Y, Jing T, Li Y, Xiao Y, Wang B, Wang W, Zhang $J$ and Lin R: SIRT1 inhibition promotes atherosclerosis through impaired autophagy. Oncotarget 8: 51447-51461, 2017.

27. Ross R: Atherosclerosis-an inflammatory disease. N Engl J Med 340: 115-126, 1999.

28. Nagy L, Tontonoz P, Alvarez JG, Chen H and Evans RM: Oxidized LDL regulates macrophage gene expression through ligand activation of PPARgamma. Cell 93: 229-240, 1998.

29. Han Y, Ma J, Wang J and Wang L: Silencing of H19 inhibits the adipogenesis and inflammation response in ox-LDL-treatedRaw264.7 cells by up-regulating miR-130b. Mol Immunol 93: 107-114, 2018.

30. Tsui L and Wang IJ: Analysis and quantification of oxidized low-density lipoprotein-induced lipid droplets in macrophages through high-content screening: Application for antiatherogenic drugs discovery. Assay Drug Dev Technol 17: 223-230, 2019.

31. Du XJ, Lu JM and Sha Y: MiR-181a inhibits vascular inflammation induced by ox-LDL via targeting TLR4 in human macrophages. J Cell Physiol 233: 6996-7003, 2018.

32. Livak KJ and Schmittgen TD: Analysis of relative gene expression data using real-time quantitative PCR and the 2(-Delta Delta C(T)) method. Methods 25: 402-408, 2001. 
33. Herrington $\mathrm{W}$, Lacey $\mathrm{B}$, Sherliker $\mathrm{P}$, Armitage $\mathrm{J}$ and Lewington $\mathrm{S}$ : Epidemiology of atherosclerosis and the potential to reduce the global burden of atherothrombotic disease. Circ Res 118: 535-546, 2016.

34. Hansson GK: Inflammation, atherosclerosis, and coronary artery disease. N Engl J Med 352: 1685-1695, 2005.

35. Ryo A, Suizu F, Yoshida Y, Perrem K, Liou YC, Wulf G, Rottapel R, Yamaoka S and Lu KP: Regulation of NF-kappaB signaling by Pin1-dependent prolyl isomerization and ubiquitin-mediated proteolysis of p65/RelA. Mol Cell 12: 1413-1426, 2003.

36. Imanishi $\mathrm{T}$ and Akasaka $\mathrm{T}$ : Novel strategies to target inflammatory processes in atherosclerosis. Curr Pharm Design 19: $1616-1625,2013$

37. LiP,Zhong X,Li J,LiuH,MaX,HeR andZhao Y: MicroRNA-30c-5p inhibits NLRP3 inflammasome-mediated endothelial cell pyroptosis through FOXO3 down-regulation in atherosclerosis. Biochem Biophys Res Commun 503: 2833-2844, 2018

38. Wei Y, Nazari-Jahantigh M, Chan L, Zhu M, Heyll K, Corbalán-Campos J, Hartmann P, Thiemann A, Weber C and Schober A: The microRNA-342-5p fosters inflammatory macrophage activation through an Akt1- and microRNA-155-dependent pathway during atherosclerosis. Circulation 127: 1609-1619,2013.

39. Zernecke A, Bidzhekov K, Noels H, Shagdarsuren E, Gan L, Denecke B, Hristov M, Köppel T, Jahantigh MN, Lutgens E, et al: Delivery of microRNA-126 by apoptotic bodies induces CXCL12-dependent vascular protection. Sci Signal 2: ra81, 2009.

40. Zhang Y, Liu D, Chen X, Li J, Li L, Bian Z, Sun F, Lu J, Yin Y, Cai $\mathrm{X}$, et al: Secreted monocytic miR-150 enhances targeted endothelial cell migration. Mol Cell 39: 133-144, 2010.
41. Nazari-Jahantigh M, Wei Y, Noels H, Akhtar S, Zhou Z, Koenen RR, Heyll K, Gremse F, Kiessling F, Grommes J, et al: MicroRNA-155 promotes atherosclerosis by repressing Bcl6 in macrophages. J Clin Invest 122: 4190-4202, 2012.

42. Qin B, Shu Y, Long L, Li H, Men X, Feng L, Yang H and Lu Z: MicroRNA-142-3p induces atherosclerosis-associated endothelial cell apoptosis by directly targeting rictor. Cell Physiol Biochem 47: 1589-1603, 2018.

43. Wu Y, Xin D, Liu C and Wang F: SIRT1 participates in epithelial-mesenchymal transition of EC-9706 and Eca-109 cells in vitro by regulating Snail expression. Nan Fang Yi Ke Da Xue Xue Bao 38: 1325-1330, 2018 (In Chinese).

44. Tian Y, Ma J, Wang W, Zhang L, Xu J, Wang K and Li D: Resveratrol supplement inhibited the NF- $\kappa \mathrm{B}$ inflammation pathway through activating AMPK $\alpha$-SIRT1 pathway in mice with fatty liver. Mol Cell Biochem 422: 75-84, 2016.

45. Jung CH, Lee MJ, Kang YM, Lee YL, Seol SM, Yoon HK, Kang SW, Lee WJ and Park JY: C1q/TNF-related protein-9 inhibits cytokine-induced vascular inflammation and leukocyte adhesiveness via AMP-activated protein kinase activation in endothelial cells. Mol Cell Endocrinol 419: 235-243, 2016.

46. Zabielska MA, Borkowski T, Slominska EM and Smolenski RT: Inhibition of AMP deaminase as therapeutic target in cardiovascular pathology. Pharmacol Rep 67: 682-688, 2015.

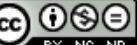

This work is licensed under a Creative Commons Attribution-NonCommercial-NoDerivatives 4.0 International (CC BY-NC-ND 4.0) License. 